\title{
Reverse Holder Condition and Space $\mathbf{A}_{\mathrm{p}}$
}

\author{
Santosh Ghimire \\ Department of Engineering Science and Humanities, Institute of Engineering \\ Pulchowk Campus, Pulchowk, Tribhuvan University, Nepal \\ Corresponding author: santoshghimire@ioe.edu.np
}

Received: Dec 25, 2017 Revised: Jan 15, 2018 Accepted: Jan 23, 2018

\begin{abstract}
In this paper, we begin with Reverse Holder condition and class $\mathrm{A}_{\mathrm{p}}$. We show that a weight function $w$ is in Reverse Holder condition $R H_{p}(d x)$ if and only if the inverse weight function $w^{-1}$ is in class $A_{p}(w d x)$. Also we show that the weight function $\mathrm{w}$ is in $A_{p}(d x)$ if and only if the inverse weight function $w^{-1}$ is in Reverse Holder condition $R H_{p^{\prime}}(d x)$.
\end{abstract}

Keywords: weight function, measure, Reverse Holder condition.

\section{Introduction}

We begin with some definitions and result which will be used in the proof of our result.

Definition: A locally integrable function on $\mathrm{R}^{\mathrm{n}}$ that takes values in the interval $(0, \infty)$ almost everywhere is called a weight. So by definition a weight function can be zero or infinity only on a set whose Lebesgue measure is zero.

We use the notation $w(E)=\int_{E} w(x) d x$ to denote the w-measure of the set $\mathrm{E}$ and we reserve the notation $L^{\mathrm{p}}\left(\mathrm{R}^{\mathrm{n}}, \mathrm{w}\right)$ or $\mathrm{L}^{\mathrm{p}}(\mathrm{w})$ for the weighted $\mathrm{L}^{\mathrm{p}}$ spaces. We note that $\mathrm{w}(\mathrm{E})<\infty$ for all sets $\mathrm{E}$ contained in some ball since the weights are locally integrable functions.

Definition: A function $\mathrm{w}(\mathrm{x}) \geq 0$ is called an $\mathrm{A}_{1}$ weight if there is a constant $\mathrm{C}_{1}>0$ such that

$$
\mathrm{M}(\mathrm{w})(\mathrm{x}) \leq \mathrm{C}_{1} \mathrm{w}(\mathrm{x})
$$

where $\mathrm{M}(\mathrm{w})$ is uncentered Hardy-Littlewood Maximal function given by

$$
M(w)(x)=\sup _{x \in B} \frac{1}{|B|} \int_{B} w(t) d t
$$

Definition: Let $1<\mathrm{p}<\infty$. A weight $\mathrm{w}$ is said to be of class $\mathrm{A}_{\mathrm{p}}$ if $[w]_{A_{p}}$ is finite where $[w]_{A_{p}}$ is defined as 


$$
[w]_{A_{p}}=\sup _{Q \text { cubes in } R^{n}}\left(\frac{1}{|Q|} \int_{Q}|w(x)| d x\right)\left(\frac{1}{|Q|} \int_{Q}|w(x)|^{\frac{-1}{p-1}} d x\right)^{p-1} .
$$

We note that in the above definition of $\mathrm{A}_{\mathrm{p}}$ one can also use set of all balls in $\mathrm{R}^{\mathrm{n}}$ instead of all cubes in $R^{n}$. Readers are suggested to read [4] for motivation, properties of $A_{p}$ weights and much more about the $A_{p}$ weights. Also refer [2] and [3] for more properties on $A_{1}$ and $A_{p}$ weight function.

\section{Reverse Holder Condition}

Let $1<\mathrm{q}<\infty$ and $\mu$ a positive measure on $\boldsymbol{R}^{n}$. We say that a positive function $\mathrm{K}$ on $\boldsymbol{R}^{n}$ satisfies a reverse Holder condition of order q with respect to measure $\mu$ if

$$
[K]_{R H_{q}(\mu)}=\sup _{Q \text { cubes in } R^{n}} \frac{\left(\frac{1}{\mu(Q)} \int_{Q} K^{q} d \mu\right)^{\frac{1}{q}}}{\frac{1}{\mu(Q)} \int_{Q} K d \mu}<\infty
$$

where the supremum is taken on all cubes Q in $\boldsymbol{R}^{n}$.

Symbolically, we write $K \in R H_{q}(\mu)$.

We now state and prove our main result.

$A$ weight function $w$ is in Reverse Holder condition $R H_{p^{\prime}}(d x)$ if and only if the inverse weight function $w^{-1}$ is in class $A_{p}(w d x)$. Also a weight function $w$ is in $A_{p}(d x)$ if and only if the inverse weight function $w^{-1}$ is in Reverse Holder condition $R H_{p}{ }^{\prime}(d x)$. Moreover, if a positive function $k$ lies in $R H_{p}(d x)$ for some $1<p<\infty$, then there exists $\delta>0$ such that $k$ lies in $R H_{p+\delta}(d x)$.

Here $p$ and $p^{\prime}$ are conjugate of each other. So we have

$$
p^{\prime}=1-\frac{1}{1-p}, 1-p^{\prime}=\frac{1}{1-p}, \quad \frac{1}{p^{\prime}}=\frac{p-1}{p}
$$

Let us write $\int_{Q} u d x=U(Q)$ and $\int_{Q} v d x=V(Q)$.

With this notations we have,

$$
I(Q):=\frac{\left(\frac{1}{U(Q)} \int_{Q}\left(v u^{-1}\right)^{p^{\prime}} u d x\right)^{\frac{1}{p^{\prime}}}}{\frac{1}{U(Q)} \int_{Q}\left(v u^{-1}\right) u d x}
$$


124 Reverse Holder Condition and Space $A_{p}$

$$
\begin{gathered}
=\frac{\left(\frac{V(Q)}{U(Q)}\right)^{\frac{1}{p}}\left(\frac{1}{V(Q)} \int_{Q} v^{p^{\prime}} u^{1-p^{\prime}} d x\right)^{\frac{1}{p^{\prime}}}}{\frac{1}{U(Q)} \int_{Q} v d x} \\
=\frac{\left(\frac{V(Q)}{U(Q)}\right)^{\frac{p-1}{p}}\left(\frac{1}{V(Q)} \int_{Q} v^{1-\frac{1}{1-p}} u^{\frac{1}{1-p}} d x\right)^{\frac{p-1}{p}}}{\frac{V(Q)}{U(Q)}} \\
=\left(\frac{V(Q)}{U(Q)}\right)^{\frac{p-1}{p}-1}\left(\frac{1}{V(Q)} \int_{Q}\left(u v^{-1}\right)^{\frac{1}{1-p}} v d x\right)^{\frac{p-1}{p}} \\
=\left[\left(\frac{1}{V(Q)} \int_{Q}\left(u v^{-1} v\right) d x\right)\left(\frac{1}{V(Q)} \int_{Q}\left(u v^{-1}\right)^{\frac{1}{1-p}} v d x\right)^{p-1}\right]^{\frac{1}{p}}:=J(Q)^{\frac{1}{p}}
\end{gathered}
$$

Note that in the above derivation the following identity was used:

$$
\left(\frac{V(Q)}{U(Q)}\right)^{\frac{p-1}{p}-1}=\left(\frac{V(Q)}{U(Q)}\right)^{\frac{1}{p}}=\left(\frac{1}{V(Q)} \int_{Q}\left(u v^{-1}\right) \quad v d x\right)^{\frac{1}{p}}
$$

Thus we have $I(Q)=J(Q)^{\frac{1}{p}}$. Now

$\left[v u^{-1}\right]_{R H_{p}{ }^{\prime}(u d x)}=\sup \frac{\left(\frac{1}{U(Q)} \int_{Q}\left(v u^{-1}\right)^{p^{\prime}} u d x\right)^{\frac{1}{p^{+}}}}{\frac{1}{U(Q)} \int_{Q}\left(v u^{-1}\right) u d x}=\sup I(Q)=\sup J(Q)^{\frac{1}{p}}=\left[u v^{-1}\right]_{A_{p}(v d x)}^{\frac{1}{p}}$

where supremum is taken over all cubes $\mathrm{Q}$ in $\boldsymbol{R}^{n}$. Let us set $u=1, v=w$, and $v=1, v=w$, we have

$$
\begin{gathered}
{[w]_{R H_{p}{ }^{\prime}(u d x)}=\left[w^{-1}\right]_{A_{p}(w d x)}^{\frac{1}{p}}} \\
{\left[w^{-1}\right]_{R H_{p^{\prime}}(u d x)}=[w]_{A_{p}(d x)}^{\frac{1}{p}}}
\end{gathered}
$$

This shows that a weight $\mathrm{w}$ is in Reverse Holder condition $R H_{p}{ }^{\prime}(d x)$ if and only if the inverse weight function $w^{-1}$ is in class $A_{p}(w d x)$. Moreover, a weight function $\mathrm{w}$ is in $A_{p}(d x)$ if and only if the inverse weight function $w^{-1}$ is in Reverse Holder condition $R H_{p}{ }^{\prime}(d x)$. 


\section{Conclusion}

We established relation between reverse Holder condition and $A_{p}$ class.

\section{References}

[1] Ba nelos R and Moore CN (1991), Probabilistic Behavior of Harmonic Functions, Birkhauser Verlag.

[2] Ghimire S (2014), Weighted Inequality, Journal of Institute of Engineering, 10(1) : 121-124.

[3] Ghimire S (2014), Two Different Ways to Show a Function is an $\mathrm{A}_{1}$ Weight Function, The Nepali Mathematical Sciences Report, 33(1\&2) : 15-19.

[4] Grafakos L (2009), Modern Fourier Analysis, Second Edition, Springer. 